\title{
Uveitis associated juvenile idiopathic arthritis - presentation of recently published international recommendations for diagnostic and therapeutic procedures
}

\author{
Lidia Rutkowska-Sak, Piotr Gietka \\ Developmental Age Rheumatology Clinic and Polyclinic, National Institute of Geriatrics, Rheumatology, and Rehabilitation, \\ Warsaw, Poland
}

\begin{abstract}
Uveitis is one of the most common extra-articular manifestations in juvenile idiopathic arthritis (JIA) and occurs in 20-30\% of children with this disease. Uveitis occurs at various frequencies and varies in character in individual clinical subtypes of the disease. Normally it is asymptomatic or mildly symptomatic; thus, in $85 \%$ of the patients the first symptoms of uveitis and its subsequent episodes pass unobserved. In $75 \%$ of cases inflammation affects both eyes. Most often it occurs in the oligoarticular subtypes of JIA, especially in patients aged 2-4 years, who are diagnosed with anti-nuclear antibodies in serum. Uncontrolled uveitis may lead to severe complications that may result in vision loss.

To date, there are no generally accepted diagnostic and therapeutic standards for this disease. This article presents the latest recommendations by ophthalmologists and rheumatologists for the detection, treatment, and monitoring of children with JIA-associated uveitis.
\end{abstract}

Key words: juvenile idiopathic arthritis, uveitis, autoimmune and autoinflammatory process.

\section{Introduction}

Autoimmune and autoinflammatory process development underlies the pathogenesis of juvenile idiopathic arthritis (JIA), the most common type of arthritis at developmental age. A chronic inflammatory process may result in extra-articular symptoms and systemic complications. The incidence of JIA in Europe varies from 1.6 to 23, and prevalence from 3.8 cases per 400/100,000 [1].

Diagnosis requires adherence to several criteria: time of onset of the disease, age $<16$ years, at least six months' duration of symptoms, and exclusion of other causes of arthritis. Exclusion list includes the following: infection-related, reactive, allergic and toxic arthritis, neoplastic, metabolic, autoimmune, and blood diseases, as well as other inflammatory and non-inflammatory connective tissue diseases. Also, psychogenic diseases and fibromyalgia are JIA exclusions [2]. Juvenile idiopathic arthritis has several clinical subtypes, which are presented in Table I.
The possibility of a varied course of the disease, especially in the first six months, scant information resulting from immunological tests, and the need to analyse all exclusions can hinder the diagnosis. Despite using numerous innovative therapies, the effect of treatment is not always satisfactory. Moreover, even 30 years after disease onset, in almost $40 \%$ of patients the disease remains constantly active [3, 4].

Uveitis (UV) is the most common extra-articular symptom of JIA. It was first observed by Hutchinson [5] and described in his article in 1873, and later by Ohm in 1910 [6]. In 1966 the clinicians from Institute of Rheumatology in Warsaw (Pietrowa, an ophthalmologist; Sowińska and Strzelecka, paediatricians) described their observations on patients with JIA-associated UV [7].

The incidence of UA refers to 4.3 out of 100,000 children, and its prevalence is 27.9 out of 100,000 children [8]. According to various authors, UA occurs in 15-67\% of cases of JIA [8]. In the course of JIA, UV develops in $20-30 \%$ of patients, most often in $4-7$ years from the 
Table I. The prevalence of various clinical subtypes of juvenile idiopathic arthritis (JIA)

\begin{tabular}{|lcc|}
\hline JIA subtypes & $\begin{array}{c}\text { The most common } \\
\text { age at disease } \\
\text { onset (years) }\end{array}$ & $\begin{array}{c}\text { Prevalence } \\
\text { (\%) }\end{array}$ \\
\hline $\begin{array}{l}\text { Oligoarticular } \\
\text { (persistent or extended) }\end{array}$ & Age 2-4 & $40-50$ \\
\hline Polyarticular, RF (-) & Age 2-4, 9-11 & $25-30$ \\
\hline Generalised & Unspecified & $13-26$ \\
\hline Polyarticular, RF (+) & Older children & $2-7$ \\
\hline $\begin{array}{l}\text { Enthesitis-related } \\
\text { arthritis }\end{array}$ & Older children & $5-8$ \\
\hline Psoriatic arthritis & Age 2-4, 9-11 & $5-8$ \\
\hline Undifferentiated & - & $11-21$ \\
\hline
\end{tabular}

occurrence of articular symptoms. However, this is not a rule because in about 3-10\% of children with JIA UV precedes arthritis [8]. Severe and recurrent UV can cause complications such as glaucoma, cataract, optic nerve damage, retinal detachment, or even loss of vision. Most often, especially in patients with the presence of anti-nuclear antibodies (ANA), JIA has an oligoarticular subtype.

The main IA-UV peak incidence is at the age of 2-4 years. Problems with verbal communication obvious at this age and the course of the disease (painless ocular lesions, not visible at first) comprise $85 \%$ of the first uveitis symptoms, and its subsequent episodes are unnoticed. Due to this limitation, at the moment of initial UV diagnosis, significant vision loss is observed in 25-33\% of children with JIA [8].

Clinically, JIA-associated uveitis is chronic non-granulomatous inflammation of the anterior section of the uvea (anterior uveitis - AUV). The inflammation of anterior part includes iridocyclitis and iritis (anterior chamber and iris). Uveitis may cause severe symptoms such as eye redness, pain, and visual disturbances with blurred vision and sensitivity to light. During slit lamp examination, a "sprinkle" pattern, small and medium precipitates on the endothelial surface, cells and exudation in anterior chamber, and dilated pupil vessels are visible. In the course of a long-term inflammatory process, inflammatory cells in vitreous humour, pigment deposits on a lens, posterior adhesions, and synechia occur. In $75 \%$ of cases inflammation affects both eyes.

Based on the analysis of the course of the disease, in over 100 children with JIA and uveitis, it was shown that the disease was more common in girls, but it was significantly more acute in boys, sometimes with elevated classical laboratory markers of acute inflammation. Also in boys, uveitis preceded other clinical symptoms of JIA more often. In the observed children, uveitis affected one eye mainly in the oligoarticular subtype of JIA, in the remaining subtypes it affected both eyes. In children with uveitis, often despite laboratory markers of acute inflammation such as C-reactive protein, erythrocyte sedimentation rate, and elevated number of thrombocytes, an elevated level of interleukin 1 was observed. The consequences of late diagnosis of uveitis were often irreversible and could lead to severe disability even in the form of eyeball atrophy $[9,10]$.

For years there were no generally accepted diagnostic and therapeutic standards for diagnostics and treatment of JIA associated uveitis.

The first recommendations for diagnostics and treatment of JIA UV were first put forward by the experts of German Scientific Society of Paediatric and Adult Ophthalmologists and Rheumatologists in 2004, subsequent updates were presented in 2007, and the last update is from 2011 [11].

\section{A.}

In recent years, as a part of the Paediatric Rheumatology International Trials Organisation (PRINTO), the Single Hub and Access point for Paediatric Rheumatology in Europe (SHARE) project has been created. The main goal of this project is to improve the care of paediatric patients suffering from rheumatic disorders.

As a part of the SHARE project, recommendations for diagnostics and treatment of UV JIA were developed on the basis of the consensus arrived at by a team of paediatric ophthalmologists and rheumatologists [12]. These recommendations are presented in Table II.

B.

In 2019, a year after the recommendations for diagnosing, monitoring, and treatment of JIA-associated uveitis of the SHARE project had been released, the guidelines from the American College of Rheumatology (ACR) and Arthritis Foundation 2019 were published [13].

The Guideline for the Screening, Monitoring, and Treatment of Juvenile Idiopathic Arthritis-Associated Uveitis of American College of Rheumatology/Arthritis Foundation [13] are presented below:

\section{Recommendations for ophthalmic examinations in} children with JIA:

1. With a high risk of development of chronic uveitis: - ophthalmic examination every 3 months.

\section{Recommendations for monitoring of ophthalmic examinations in children with JIA and controlled chronic uveitis:}

1. During exchange, reduction, and after completion of topical treatment with glucocorticosteroids:

- ophthalmic examination every month. 
Table II. Summarised points of consensus-based recommendations for the management of uveitis associated with juvenile idiopathic arthritis (JIA): the SHARE initiative [11]

\begin{tabular}{|c|}
\hline \\
\hline $\begin{array}{l}\text { 1. All children with JIA should be tested for uveitis } \\
\text { 2. Frequency of follow-up visits should be scheduled on the basis of the course of the disease and agreed to by an ophthalmologist } \\
\text { 3. Children in whom immunosuppressive treatment was withdrawn are susceptible to a new episode or recurrence of uveitis. } \\
\text { After immunosuppressive treatment is completed, all the children should undergo ophthalmic examination every } 3 \text { months } \\
\text { for at least } 1 \text { year }\end{array}$ \\
\hline \\
\hline $\begin{array}{l}\text { 1. There is a need for close cooperation between an ophthalmologist and a paediatric rheumatologist in the area of modification } \\
\text { of treatment, depending on the activity of the disease } \\
\text { 2. There is a need to develop generally acceptable criteria for assessment of uveitis activity helpful in the selection of systemic } \\
\text { therapy } \\
\text { 3. Currently, there is no biomarker agreed upon that would monitor uveitis activity } \\
\text { 4. Currently, there is no widely accepted definition of inactive eye disease in the course of IIA. Cellularity in the anterior chamber } \\
\text { should not be the goal of treatment of uveitis. The presence of macular oedema and/or papilledema, lowering of intraocular } \\
\text { pressure, and rubeosis iridis may require anti-inflammatory treatment despite the lack of cells in the anterior chamber } \\
\text { 5. A 2-year therapy with classic or biologic modifying drugs right after a local therapy with glucocorticosteroids is recommended }\end{array}$ \\
\hline \\
\hline $\begin{array}{l}\text { 1. Active uveitis requires immediate treatment } \\
\text { 2. Topical glucocorticosteroids (preferably prednisolone acetate or dexamethasone) are first-line drugs in uveitis therapy } \\
\text { 3. Topical or general nonsteroidal anti-inflammatory drugs are not proven to be efficient in monotherapy, but they may be } \\
\text { used as supplementary drugs } \\
\text { 4. During the first visit a systemic immunosuppressive therapy for active chronic uveitis is recommended in case factors } \\
\text { of bad prognosis are present. The presence of factors of bad prognosis, including the lack of remission in later phase } \\
\text { of the disease, also requires systemic immunosuppression } \\
\text { 5. Systemic immunosuppression is recommended in case no improvement in topical treatment is obtained for } 3 \text { months } \\
\text { or in case uveitis relapses during reduction of doses glucocorticosteroids } \\
\text { 6. Methotrexate has been the drug of choice in systemic immunosuppression } \\
\text { 7. In case methotrexate is not effective or not tolerated, it is recommended to include a biologic drug or switch to a biologic drug } \\
\text { 8. Use of anti-TNF drugs (adalimumab > infliximab > golimumab) is recommended in patients who are resistant to modifying } \\
\text { drugs, especially to methotrexate } \\
\text { 9. On the basis of current evidence, etanercept should not be take into consideration as a therapy for JIA-associated uveitis } \\
\text { 10. Exchanging various anti-TNF drugs is not recommended in the event that the first used anti-TNF drug is ineffective, even } \\
\text { if current evidence is based on the results of research on small groups of patients } \\
\text { 11. In case of further ineffectiveness of treatment, a test for the presence of antibodies against the used drugs or their low } \\
\text { concentration should be considered. With the lack of antibodies and/or low concentration of the used drug, increasing } \\
\text { the doses or shortening the time interval between individual doses should be taken into consideration } \\
\text { 12. In cases of resistance to the used TNF inhibitors, treatment with tocilizumab, rituximab, or abatacept should be considered } \\
\text { as an option }\end{array}$ \\
\hline
\end{tabular}

2. During stable treatment:

- ophthalmic examination every 3 months.

3. During reduction or withdrawal of systemic therapy:

- ophthalmic examination every 2 months.

III. Recommendations for the use of glucocorticosteroids:

In children and adolescents with JIA and active chronic uveitis the following is recommended:

1. Focal use of $1 \%$ prednisolone acetate (when compared to other topical drugs).

2. Introduction or increase in doses of topical glucocorticosteroids for short-term control of new or relapsed inflammation in the eye instead of introducing systemic glucocorticosteroids. However, in patients who require 1-2 drops of $1 \%$ prednisolone acetate (or 1\% equivalent) to control uveitis if they do not get systemic therapy, introduction of systemic therapy and maintaining focal glucocorticosteroids is recommended. If the patients still require $1-2$ drops of $1 \%$ prednisolone acetate or (1\% equivalent) with at least three-month long use of systemic treatment, a change or intensification of systemic treatment is recommended.

IV. Recommendations for classic and biologic DMARDs (disease-modifying antirheumatic drugs) in children and adolescents with JIA and chronic uveitis:

1. At the start of treatment, subcutaneous administration of methotrexate is recommended because it is more effective when compared to oral form. 
2. In case of severe uveitis, posing a threat of complications, initiation of methotrexate therapy with TNF inhibitor in the form of a monoclonal antibody, and not a receptor, is recommended.

3. In the event of insufficient response to one TNFi monoclonal antibody, increase in the dose or the frequency of administration, and if there is still no improvement, a change of the type of antibody is recommended.

4. In patients who did not respond to methotrexate and subsequently to two monoclonal antibodies TNFi at abatacept or tocilizumab are recommended as biological options of DMARDs, and mycophenolate, leflunomide or ciclosporin, as alternative, non-biological options of DMARDs.

\section{Recommendations for withdrawal of therapy in JIA} with chronic uveitis:

1. In children with the disease that is controlled with systemic therapy but that requires administration of one to two drops of $1 \%$ prednisolone acetate a day, reduction of topical, ocular glucocorticosteroids doses is strongly recommended before reduction of doses of the drugs administered systemically.

2. In patients with uveitis that is well controlled only with classic and/or biologic DMARDs administered systemically, the therapy should be withdrawn after at least wot years of the disease being well controlled.

\section{C.}

Also last year, eight paediatric rheumatologists and seven ophthalmologists, as the Multinational Interdisciplinary Working Group for Uveitis in Childhood (MIWGUC), published a proposal of definitions concerning: response to treatment, inactive disease, and damage for IIA-associated uveitis (juvenile idiopathic arthritis-associated uveitis - JIAU) based on the validation of outcomes of assessment [14]. According to the doctors associated in MIWGUC, the assessment should be carried out jointly by a paediatric ophthalmologist, rheumatologist, and the patient or patient's parent.

The main proposals of MIWGUC [14]:

\section{Assessment by an ophthalmologist:}

1. Anatomical location of the inflammatory process.

2. Activity of inflammatory lesions according to SUN (Standardisation of Uveitis Nomenclature), grades $0-4$, depending on the number of inflammatory cells within a field sized $1 \times 1 \mathrm{~mm}$ of biomicroscope [0 (< 1), 0.5+ (1-5), 1+ (6-15), 2+ (16-25), 3+ (26-50), $4+(>50)$ ] or according to MIWGUC (only four grades) and a VAS scale (0-100 $\mathrm{mm})$.

3. Visual acuity documented with a chart with $\log M A R$.

4. Eye fundus examination.

5. Laser photometry, if available.

6. Presence of structural complications:
- ocular hypotony (IOP $\leq 6 \mathrm{~mm} \mathrm{Hg})$,

- ocular hypertension ( $\geq 21 \mathrm{~mm} \mathrm{Hg}$ ),

- posterior adhesions,

- glaucoma,

- cataract,

- band keratopathy,

- papilloedema,

- macular oedema,

- epiretinal membrane,

- floaters.

7. Significant weakness of sight $(<0.3)$.

\section{Assessment by a paediatrician-rheumatologist:}

1. Age at onset of the disease and its duration.

2. Number of inflammatory active joints.

3. Type of treatment.

4. General activity of the disease on a VAS scale (0-100 mm).

\section{Assessment by a patient/parent:}

1. Number of days spent at a hospital and school/ kindergarten absences.

2. Limitations in everyday activities in the last six months on a CHAQ scale (0-3).

3. Quality of life on PedsQL scale (0-100).

4. General well-being on a VAS scale $(0-100 \mathrm{~mm})$.

5 . Response to treatment.

\section{Remission in juvenile idiopathic arthritis uveitis}

With reference to Wallace's criteria for IIA, a remission in JIAU during and without pharmacotherapy is defined by meeting the following conditions after at least six months of treatment or during inactive eye inflammatory disease for more than three months after all anti-inflammatory therapies used to treat uveitis were completed:

1. Total number of AC cells in a slit lamp - 0 of inflammatory cells, in patients with apnoea individual cells may be present.

2. No papilloedema.

3. No macular oedema.

4. No floaters $(<0.5+)$.

5. No other complications.

6. General assessment by an ophthalmologist of the grade of activity of uveitis on a VAS scale on the range from 0 to $100 \mathrm{~mm}$ must be 0 .

7. General assessment by a rheumatologist of the grade of disability due to uveitis on a VAS scale on the range from 0 to $100 \mathrm{~mm}$ must be 0 .

8. General assessment by a patient/parent of the grade of disability due to uveitis on a VAS scale on the range from 0 to $100 \mathrm{~mm}$ must be 0 .

9. Always both eyes must be assessed. 
What is consistent in the presented recommendations is the need for ophthalmological control also in asymptomatic (in terms of UV) patients with $\mathrm{JA}$, more frequent eye examination in case of diagnosed UV, and consent of specialists is visible in subject of necessity of both eye assessment.

\section{Conclusions}

Following the recommendations and proposals of paediatricians and rheumatologists, the common approach to patients with JIA and uveitis should be special, because complications of JIA can result in severe and permanent disability, and points to a worse course of this autoimmune disease.

The message for rheumatologists results, among others, from the SHARE initiative and ACR/Arthritis Foundation guidelines, and is that severe JIA-associated uveitis requires combined therapy with a biological drug. This procedure allows the control of UV activity and avoidance of complications. Therefore, it should be stressed that children with severe uveitis, who are non-responsive for treatment with DMARDs for 12 weeks, irrespective of the number of affected joints, may be qualified to the treatment with biologics.

In addition, the recommendations prepared in recent years, collected in the presented article, facilitate argumentation to apply early enough more aggressive and effective treatment of this group of patients.

The authors declare no conflict of interest.

\section{References}

1. Thierry S, Fautrel B, Lemelle I, Guillemin F. Prevalence and incidence of juvenile idiopathic arthritis: a systematic review. Joint Bone Spine 2014; 81: 112-117, DOI: 10.1016/j.jbspin.2013. 09.003.

2. Cassidy JT, Petty RE. Juvenile idiopathic arthritis. In: Textbook of pediatric rheumatology, 5th ed., Cassidy JT, Petty RE (eds.). WB Saunders, Philadelphia 2005; 291-303.

3. Packham JC, Hall MA. Long-term follow-up of 246 adults with juvenile idiopathic arthritis: functional outcome. Rheumatology (Oxford) 2002; 41: 1428-1435, DOI: 10.1093/rheumatology/41. 12.1428.
4. Selvaag AM, Aulie HA, Lilleby V, Flatø B. Disease progression into adulthood and predictors of long-term active disease in juvenile idiopathic arthritis. Ann Rheum Dis 2016; 75: 190-195, DOI: 10.1136/annrheumdis-2014-206034.

5. Hutchinson J. On a peculiar form of iritis which occurs in the children of gouty parents. Lancet 1-3, 1873.

6. Ohm J. Bandfoermige Hornhauttrübung bei einem neunjaehrigen Mädchen und ihre Behandlung mit subconjunctivalen Jodkaliumeinsprizungen. Klin Monatsbl Augenheilkd 1910; 48: 243 (acc. Petty RE. Current knowledge of the etiology and pathogenesisof chronic uveitis accompanying juvenile rheumatoid arthritis. Rheum Dis Clin North Am 1987; 13: 19-36).

7. Pietrowa N, Sowińska J, Strzelecka M. Early ophtalmic changes as the first manifestation of rheumatoid arthritis in children. Reumatologia 1966; 4: 305-309 [In Polish].

8. Clarke SLN, Ethan S, Ramanan V. Juvenile idiopathic arthritisassociated uveitis. Pediatr Rheumatol Online J 2016; 14: 27, DOI: 10.1186/s12969-016-0088-2.

9. Vitale AT, Graham E, de Boer JH. Juvenile idiopathic arthritisassociated uveitis: clinical features and complications, risk factors for severe course, and visual outcome. Ocul Immunol Inflamm2013;21:478-485,DOI:10.3109/09273948.2013.815785.

10. Rutkowska-Sak L, Stawowski R. Zapalenie błony naczyniowej oka w przebiegu młodzieńczego idiopatycznego zapalenia stawów. Reumatologia 2012; 50 (supl. 1).

11. Heiligenhaus A, Michels $H$, Schumacher $C$, et al. Evidencebased, interdisciplinary guidelines for anti-inflammatory treatment of uveitis associated with juvenile idiopathic arthritis. Rheumatol Int 2012; 32: 1121-1133, DOI: 10.1007/s00296011-2126-1.

12. Constantin T, Foeldvari I, Anton J, et al. Consensus-based recommendations for the management of uveitis associated with juvenile idiopathic arthritis: the SHARE initiative. Ann Rheum Dis 2018; 77: 1107-1117, DOI: 10.1136/annrheumdis2018-213131.

13. Angeles-Han ST, Ringold S, Beukelman T, et al. 2019 American College of Rheumatology/Arthritis Foundation Guideline for the screening, monitoring, and treatment of juvenile idiopathic-associated uveitis. Arthritis Care Res (Hoboken) 2019; 71: 703-713, DOI: 10.1002/acr.23871.

14. Foeldvari I, Klotsche J, Simonini G, et al. Proposal for a definition for response to treatment, inactive disease and damage for JIA associated uveitis based on the validation of a uveitis related JIA outcome measures from the Multinational Interdisciplinary Working Group for Uveitis in Childhood (MIWGUC). Pediatr Rheumatol Online J 2019; 17: 66, DOI: 10.1186/s12969019-0345-2. 